\title{
Recovery from sedation during regional anesthesia
}

\author{
Tae-Yop Kim \\ Department of Anesthesiology, Konkuk University Medical Center, Konkuk University School of Medicine, Seoul, Korea
}

\section{Introduction}

The use of regional anesthesia has been increasing for various surgical procedures. Sedation is required to promote patient comfort and to avoid painful emotional and physical stimuli. In the case of inadequate analgesia even after regional anesthesia, sedation may be supplemented by an opioid or a non-opioid analgesic to improve surgical condition. Various intravenous hypnotic agents can be used for this purpose as in conscious sedation or "monitored anesthesia care (MAC)" for diagnostic and interventional procedures; the centrally active intravenous hypnotic agents such as benzodiazepines and propofol are preferred to provide optimum patient sedation. Additionally, an analgesia-based technique employing ultrashort acting remifentanil has also been used to ensure patient comfort with or without administering a hypnotic agent in the intensive care unit. Dexmedetomidine, a selective alpha-2 agonist, has recently been widely used for sedation in various clinical settings [1]. The choice and titration of dosage should be carefully planned by responding to a patient's status and type of surgery, and the degree of sedation should be maintained to allow the patient to communicate with anesthesiologists throughout the entire surgical procedure [2].

While understanding the pharmacokinetic and pharmacodynamic effects of commonly used sedative and analgesic drugs is necessary to provide an acceptable degree of sedation, their residual effects should be considered to avoid prolonged post-procedural awakening.

\section{Benzodiazepines}

All benzodiazepines provide varying degrees of sedation with amnesia. Benzodiazepine-induced sedation due to central nervous system depression is dose-dependent; the degree of sedation can vary from light to deep unconsciousness.

Diazepam $(0.1-0.2 \mathrm{mg} / \mathrm{kg})$ has long been used as a prototypic drug but its characteristically long elimination half-life has been a concern (about $50 \mathrm{~h}$ ); this is due to active metabolites recirculating into entero-hepatic circulation. Significant delay in recovery and postoperative re-sedation can be associated with the use of a larger dosage. The sedation effect of diazepam is particularly intensified in older patients under local anesthesia and its poor controllability to achieve desired sedative level; thus, its dosage should be carefully selected and adjusted.

Midazolam (0.05-0.1 mg/kg) is the most popular anxiolytic and hypnotic agent used in surgical and non-surgical settings. As a water soluble agent, its onset time is much faster, and it has a relatively short elimination half-life $(2-4 \mathrm{~h})$, allowing a more predictable recovery than diazepam. Therefore, midazolam is preferred for brief procedures. However, intravenous midazolam should also be tittered to achieve and maintain the desired sedative level as well as to minimize side effects due to overdosage [3].

Flumazenil can reverse the effects of benzodiazepines; thus, it is commonly employed to treat benzodiazepine overdose. Its onset of action is rapid within 1-2 min and its peak effect occurs in 6-10 $\mathrm{min}$. The recommended dose for adults is 200 $\mu \mathrm{g}$ every $1-2 \mathrm{~min}$ to a maximum of $3 \mathrm{mg} / \mathrm{h}$.

\section{Propofol}

Propofol is commonly used for conscious sedation or MAC in combination with opioid analgesics for various clinical settings. It is a short acting, and easily controllable hypnotic

Corresponding author: Tae-Yop Kim, M.D., Ph.D., Department of Anesthesiology, Konkuk University Medical Center, Konkuk University School of Medicine, 4-13, Hwayang-dong, Gwangjin-gu, Seoul 143-729, Korea. Tel: 82-2-2030-5445, Fax: 82-2-2030-5449, E-mail: taeyop@kuh.ac.kr (c) This is an open-access article distributed under the terms of the Creative Commons Attribution Non-Commercial License (http:// creativecommons.org/licenses/by-nc/3.0/), which permits unrestricted non-commercial use, distribution, and reproduction in any medium, provided the original work is properly cited. 
and sedative agent. The unique pharmacokinetic properties of propofol with rapid redistribution provides quick recovery from the effect of a single bolus administration as well as following a continuous infusion during a brief period. Excitatory phenomena or involuntary movements are also rare. However, its optimal dosage providing subhypnotic sedation for each individual is highly variable [4]. The rapid onset and recovery from propofol ensuring a patient's prompt responsiveness can be achieved by optimal titration of the infusion rate; the incidence of undesirable side-effects is regarded as relatively low at its subhypnotic dosage [5]. Therefore, despite very little cardiovascular and respiratory depressant effect at low-dose infusions of propofol [6], oxygen saturation monitoring is recommended and supplemental oxygen should be provided throughout the procedure.

Propofol as a supplemental agent for sedation during regional anesthesia (loading doses of $0.2-0.5 \mathrm{mg} / \mathrm{kg}$ and maintenance dosages of $0.5-4 \mathrm{mg} / \mathrm{kg} / \mathrm{h}$ ) [7] provides a smaller amnesic effect but a quicker recovery [8].

Target-controlled infusion (TCI) is a logical approach to administer an intravenous anesthetic agent. A TCI system for propofol, using an open three-compartment pharmocokinetic model, allows a more rapid adjustment of the blood concentration of propofol according to individual's need than that of manually controlled infusion. The target concentration of propofol of $0.4-1.0(1.2) \mu \mathrm{g} / \mathrm{ml}$ is useful for conscious sedation or MAC but is dependent on the age of the patient and the kind of premedication [9]. Sedation level is easily titrated by changing the target concentration of TCI, and the degree of sedation score (depth) can be evaluated to match the desired level within a few min.

\section{Intermediate- and Ultra-short Acting Opioids}

Opioid analgesics can be used as the sole supplement to local anesthesia. However, due to their inability to provide reliable sedation, opioids are often used in combination with sedative drugs to supplement analgesia induced by local anesthetics.

Pethidine (Demerol) is more lipid-soluble than morphine, resulting in a faster onset of action. Its duration of clinical effect is $2-4 \mathrm{~h}$ and half-life is $3-5 \mathrm{~h}$. Norpethidine (normeperidine), a metabolite of pethidine, can accumulate (half-life of 8-21 h) with use of larger dosages of pethidine in elderly patients or in patients with hepatic and renal dysfunction and is associated with toxic effects such as convulsion or myoclonus.

Intravenous fentanyl shows almost an immediate effect when the drug is administered intravenously. However, the maximal analgesic and respiratory depressant effect may not be noted for several min. Its analgesic effect persists for 30-60 min after a single intravenous dose of up to $100 \mu$ g (equivalent to 75 mg of pethidine), and its terminal elimination half-life is 219 $\min$.

Naloxone can be used to counter the effects of opiate overdose and life-threatening depression of the central venous and respiratory systems. It acts within $1 \mathrm{~min}$ and lasts up to $45 \mathrm{~min}$ if injected intravenously.

Remifentanil, metabolized by esterase, has a very short context-sensitive half-time (about $3.5 \mathrm{~min}$ ), which allows for a more precise intraoperative titration and has a more predictable offset of effect even during prolonged infusion. Infusion rates of $0.02-0.25 \mu \mathrm{g} / \mathrm{kg} / \mathrm{min}$ for conscious sedation with remifentanil are recommended for titration to an individual's needs. In particular, when combined with propofol at lower dosages, remifentanil provides superior analgesia during nerve blockade, thereby enhancing patient comfort during the surgical procedure without compromising hemodynamic stability or respiratory depression [10].

\section{Dexmedetomidine}

Dexmedetomidine has been introduced for sedation during diagnostic and therapeutic procedures. Its unique analgosedative effect is a fascinating feature contributing to its preference in various clinical settings. An intravenous infusion of dexmedetomidine is commonly initiated with a $1.0 \mu \mathrm{g} / \mathrm{kg}$ loading dose, administered over $10 \mathrm{~min}$, and followed by a maintenance infusion of $0.2-1.0 \mu \mathrm{g} / \mathrm{kg} / \mathrm{h}$. It shows great individual variability in producing a sedative effect; and its dosage must be carefully adjusted and titrated to achieve the desired clinical effect. Due to its relative long half-life (terminal elimination half-life of approximately $2 \mathrm{~h}$ ), prolonged patient recovery or a residual sedative effect may be a concern after the procedure. In particular, its dosage must be significantly reduced in patients with hepatic dysfunction. Dexmedetomidine $(1.0 \mu \mathrm{g} / \mathrm{kg}$ followed by $0.2 \mu \mathrm{g} / \mathrm{kg} / \mathrm{h}$ ) shows a longer recovery time and requires more rescue sedatives and analgesics than the combination of midazolam (1 mg) and fentanyl (1 $\mathrm{gg} / \mathrm{kg})[11]$.

\section{Conclusion}

While the need for sedation is growing in many different settings including surgical procedures under regional anesthesia and its application is exploding beyond the operating room, various intravenous hypnotics and opioid analgesics have shown distinct advantages for providing a sufficient level of sedation. However, their impact on postoperative recovery profile and appropriate post-operative strategy should be considered. 


\section{References}

1. Ok HG, Baek SH, Baik SW, Kim HK, Shin SW, Kim KH. Optimal dose of dexmedetomidine for sedation during spinal anesthesia. Korean J Anesthesiol 2013; 64: 426-31.

2. Stevens MH, White PF. Monitored anesthesia care. In: Anesthesia. 4th ed. Edited by Miller RD: New York, Churchill Livingstone. 1994, pp 1465-80.

3. White PF, Vasconez LO, Mathes SA, Way WL, Wender LA. Comparison of midazolam and diazepam for sedation during plastic surgery. Plast Reconstr Surg 1988; 81: 703-12.

4. Mackenzie N, Grant IS. Propofol for intravenous sedation. Anaesthesia 1987; 42: 3-6.

5. Rosa G, Conti G, Orsi P, D’Alessandro F, La Rosa I, Di Giuqno G, et al. Effects of low-dose propofol on central respiratory drive, gas exchanges and respiratory pattern. Acta Anaesthesiol Scand 1992; 36: 128-31.

6. Smith I, White PF, Nathanson M, Gouldson R. Propofol. An update on its clinical uses. Anesthesiology 1994; 81: 1005-43.

7. Smith I, Monk TG, White PF, Ding Y. Propofol infusion during regional anesthesia: sedative, amnestic and anxiolytic properties. Anesth Analg 1994; 79: 313-9.

8. White PF, Negus JB. Sedative infusions during local and regional anesthesia: a comparison of midazolam and propofol. J Clin Anesth 1991; 3: 32-9.

9. Casati A, Fanelli G, Casaletti E, Colnaghi E, Cedrati V, Torri G. Clinical assessment of target-controlled infusion of propofol during monitored anesthesia care. Can J Anaesth 1999; 46: 235-9.

10. Holas A, Krafft P, Marcovic M, Quehenberger F. Remifentanil, propofol or both for conscious sedation during eye surgery under regional anaesthesia. Eur J Anaesthesiol 1999; 16: 741-8.

11. Zeyneloglu P, Pirat A, Candan S, Kuyumcu S, Tekin I, Arslan G. Dexmedetomidine causes prolonged recovery when compared with midazolam/fentanyl combination in outpatient shock wave lithotripsy. Eur J Anaesthesiol 2008; 25: 961-7. 